\title{
Analytical model and spectral correction of vibration effects on Fourier Transform Spectrometer.
}

\author{
Irina Shatalina ${ }^{\mathrm{a}}$, Frederic Schmidt ${ }^{\mathrm{b}}$, Bortolino Saggin ${ }^{\mathrm{a}}$, Nicolas Gac ${ }^{\mathrm{c}}$, Matthieu Kowalski ${ }^{\mathrm{c}}$ and \\ Marco Giurannad.
}

\begin{abstract}
${ }^{a}$ Dipartimento di Meccanica, Politecnico di Milano, Campus of Lecco, via Gaetano Previati 1/c, 23900, Lecco, Italy; ${ }^{\mathrm{b}}$ Univ. Paris-Sud, Laboratoire IDES, UMR 8148, Bât 509, Orsay, F91405, France; 'Laboratoire des Signaux et Systèmes (L2S), UMR8506 Univ Paris-Sud CNRS - SUPELEC, SUPELEC, 3 rue Joliot Curie, Gif sur Yvette, F-91192, France; ${ }^{\text {dFSI, via }}$ del Fosso del Cavaliere 100, 00133, Roma, Italy
\end{abstract}

\begin{abstract}
Sensitivity to mechanical vibrations of Fourier Transform Spectrometers (FTS) is a well-known phenomenon. It is especially critical for FTS devoted to atmospheric studies (like the Planetary Fourier Spectrometer (PFS) onboard Mars Express 2003), as absorption bands for the gases of low concentration are comparable with the generated instrument spectral noise. The adopted techniques for the vibration sensitivity reduction suffer of limitations in practical implementation, leaving residual modulations of the interferogram and the so-called ghosts in the spectra. Moreover as it is often impossible to measure the vibrations during the FTS measurement, the position and magnitude of these ghosts cannot be evaluated. Up to now the adopted ghost reduction techniques are mostly based on the averaging of spectra, because the disturbance phase is randomly distributed. This paper presents an innovative data treatment technique which allows single spectrum correction from distortions of unknown nature. Such a technique would increase the spatial resolution of the mapping process and becomes crucial when the desired information is linked to a particular mapping area associated to an individual spectrum.

The full study consists in the explicit analysis of the ghost formation and the post-processing algorithm based on the semiblind deconvolution method - an iterative numerical algorithm of the series of consecutive deconvolutions. The technique was tested on the data from the PFS and the algorithm proved to be consistent according to the selected efficiency criteria (coming from the available general information about the signal spectral shape).
\end{abstract}

Keywords: PFS; Fourier Transform Spectrometer; Micro-vibration; Distortions; Spectroscopy; Analytical model; SemiBlind Inverse problem.

\section{INTRODUCTION}

Sensitivity to mechanical vibrations of Fourier Transform Spectrometers is a well-known phenomenon. Vibration generated spectral distortions are especially critical for FTS devoted to atmospheric studies, as the gas composition determination is based on the matching of spectral features deriving from absorption bands that for the low concentration element are comparable with the instrument spectral noise. The adopted techniques aiming at the reduction of the vibration sensitivity (such as the constant optical path step sampling based on reference laser signal trigger), suffer of limitations in the practical implementation: time delays in the acquisition chain and cyclical fluctuations of optical misalignments lead to residual vibration induced modulations of the interferogram and eventually to the so-called ghosts in the spectra. Moreover as it is often impossible to measure the vibrations during the FTS measurement, the position and magnitude of these ghosts cannot be evaluated. Up to now the adopted ghost reduction techniques ${ }^{1-3}$ are mostly based on the averaging of spectra, being the disturbance phase randomly distributed.

This paper presents an innovative data treatment technique able to deal with undesired spectral distortions of unknown nature allowing the single spectrum correction. Such a technique would increase the spatial resolution of the mapping 
process by avoiding the need of spectral averaging and becomes crucial when the desired information is linked to a particular mapping area associated with an individual spectrum.

As first step of the study the ghost nature and mechanisms of formation is analyzed in detail; the modulations of the signal due to the vibration generated misalignments (coming from deviations of the real instrument configuration from the ideal optical system ${ }^{4}$ ) and that due to the non-constant OPD (Optical Path Difference) sampling step caused by the phase delay between reference and main signal acquisition chains ${ }^{1,5-8}$ are modeled. The result is the explicit mathematical description of the interferogram modulations generated by the mechanical vibrations. The obtained model allows implementing the second step of the research i.e. the post-processing algorithm.

The proposed approach of vibration effect correction is based on the semi-blind quasi-deconvolution method - an iterative numerical algorithm of the series of consecutive quasi-deconvolutions. The general problem of the data post-processing was subdivided into three separate sub-problems: definition of the vibration kernel, recovering of the original spectrum from the distorted one and the results validation.

The technique has been tested on the data from the PFS (Planetary Fourier Spectrometer) onboard the Mars Express ESA (European Space Agency) mission. The PFS is a FTIRS (Fourier Transform InfraRed Spectrometer) composed by two channels: Short Wavelength Channel (SWC) for the 1.2-5 $\mu \mathrm{m}$ range and Long Wavelength Channel (LWC) for 5-45 $\mu \mathrm{m}$ one. The interferometer scheme of PFS is a modified Michelson with swinging Cubic Corner Mirrors instead of translating flat ones ${ }^{8-12}$. The problem of microvibrations is mostly referred to the SWC data ${ }^{13,14}$.

The key-point of the procedure was the kernel determination. The lack of information about the vibrations and their nature is the main difficulty: frequency, intensity and phase with respect to the main signal interferogram are only statistically known from PFS onboard self-check measurements that are performed at most once per orbit. The iterative procedure of kernel definition was performed on smoothed spectral data, each iteration loop including constrained quasideconvolutions of measured distorted spectrum with signal and kernel estimates. Proposed general constraints were the spectrum smoothness and kernel sparsity. The final deconvolution step was performed on the unsmoothed data, with the aim to retrieve the original spectrum from the distorted one.

Efficiency of this technique strongly depends on the initial guesses for the semi-blind deconvolution input. Also the correct definition of the deconvolution constraints is important. Utilization of additional information about the expected spectral features (e.g. some gas absorption bands, regions of detector low responsivity, any known vibration components, etc.) makes proposed algorithm more robust.

Finally the algorithm proved to be consistent according to the selected efficiency criteria (coming from the available general information about the signal spectral shape).

\section{ANALYTICAL DIRECT MODEL}

\subsection{Vibration effect: Direct Model, general formulation}

Mechanical disturbances modulate the interferogram measurements in two ways ${ }^{1}$ :

- $\quad$ by creating sampling step errors due to the different phase delays in the acquisition systems;

- by generating cyclic misalignments in the optical system.

In the following the mechanical disturbance effect is modeled for the case of a monochromatic radiation source and the disturbance being represented by one pure harmonic component.

The modulated part of the monochromatic source interferogram in the ideal interferometer can be expressed as ::

$$
I_{\sigma_{\mathrm{s}}}\left(x_{\mathrm{k}}\right)=m I_{\mathrm{s}} \cos \left(2 \pi \sigma_{\mathrm{s}} x_{\mathrm{k}}\right)
$$

where $x_{\mathrm{k}}$ is the OPD at the $\mathrm{k}^{\text {ith }}$ zero-crossing of the reference signal, $\sigma_{\mathrm{s}}$ is the wavenumber of the observed line and $m$ is the modulation factor referred to the misalignments of the optical system from its ideal position, $I_{\mathrm{s}}$ the source halfintensity.

The pure harmonic disturbance can be described as follows: 


$$
\mathbf{y}=\mathbf{y}_{\mathbf{d}} \sin \left(\omega_{\mathrm{d}} t+\varphi_{\mathrm{d}}\right)
$$

where $\mathbf{y}$ is the vector of the vibration component generated at the source, being $\omega_{\mathrm{d}}=2 \pi f_{\mathrm{d}}$ its angular frequency and $\varphi_{\mathrm{d}}$ its phase. The component phase generally is of a random nature with respect to single interferogram (while it is unambiguously defined for each $\omega_{\mathrm{d}}$ ), with uniform probability distribution in the $\pm \pi$ range for the whole set of measurements.

We represent the mirror speed variations and optical misalignments in the PFS in the instrument coordinate system, taking one axis along the optical axis of cube corner $(\mathrm{x})$, and the others perpendicular to it ( $\mathrm{u}$ and $\mathrm{w})$ :

$$
\begin{gathered}
\Delta x=x_{\mathrm{d}} \sin \left(\omega_{\mathrm{d}} t+\varphi^{\mathrm{x}}{ }_{\mathrm{d}}\right) \\
\Delta u=u_{\mathrm{d}} \sin \left(\omega_{\mathrm{d}} t+\varphi^{\mathrm{u} *}{ }_{\mathrm{d}}\right)
\end{gathered}
$$

The component phases $\varphi^{\mathrm{u}^{*}}{ }_{\mathrm{d}}$ and $\varphi^{\mathrm{x}}{ }_{\mathrm{d}}$ can be different, as the transfer function of vibration propagation from the source to the instrument will be in general different for each direction.

The modulation factor $m$ is a function of Cubic Corner Mirror (CCM) position error $u$ (in any direction perpendicular to its optical axis) and in the first order approximation it can be written in the following form ${ }^{1}$ :

$$
m\left(\sigma_{\mathrm{s}}, u\right) \cong m_{\mathrm{o}}\left(\sigma_{\mathrm{s}}, u_{o}\right)+\sigma_{\mathrm{s}} a\left(\sigma_{\mathrm{s}}, u_{o}\right) \cdot \sin \left(\omega_{\mathrm{d}} t+\varphi_{\mathrm{d}}^{\mathrm{u}}\right)
$$

where $a$ is proportional to the vibration amplitude $u_{\mathrm{d}}$ and depends on the wavenumber $\sigma_{\mathrm{s}}$ of the modulated component and the initial interferometer alignment quality $u_{\mathrm{o}}$ (zero for a perfectly aligned instrument).

For the vibration component along theCCM axis we have first order approximation for the sampling steps ${ }^{1}$ :

$$
x_{\mathrm{k}}=k \frac{\lambda_{\mathrm{r}}}{2}+v_{\mathrm{m}} T_{\mathrm{D}}+v_{\mathrm{o}} T_{\mathrm{D}} \cos \left(\omega_{\mathrm{d}} t_{\mathrm{k}}+\varphi_{\mathrm{d}}^{\mathrm{x}}\right)
$$

where $\mathrm{T}_{\mathrm{D}}$ is the time delay in the sampling chain, $\lambda_{\mathrm{r}}$ is the reference laser wavelength, $t_{\mathrm{k}}$ is the time of the $\mathrm{k}^{\text {th }}$ sampling and $v_{\mathrm{o}}$ is the vibration amplitude expressed as speed $\left(v_{\mathrm{o}}=\omega_{\mathrm{d}} \mathbf{X}_{\mathrm{d}}\right)$.

The interferogram now can be rewritten substituting Eq. (5) and Eq. (6) in Eq. (1):

$$
\begin{aligned}
& I_{\sigma_{\mathrm{s}}}\left(x_{\mathrm{k}}\right)=\left[m_{\mathrm{o}}\left(\sigma_{\mathrm{s}}, u_{o}\right)+a\left(\sigma_{\mathrm{s}}, u_{o}\right) \sigma_{\mathrm{s}} \sin \left(\omega_{\mathrm{d}} t_{\mathrm{k}}+\varphi^{\mathrm{u}}{ }_{\mathrm{d}}\right)\right] I_{\mathrm{s}} \\
& \times \cos \left[2 \pi \sigma_{\mathrm{s}}\left(\frac{k \lambda_{\mathrm{r}}}{2}+T_{\mathrm{D}} v_{\mathrm{m}}\right)+2 \pi \sigma_{\mathrm{s}} v_{\mathrm{o}} T_{\mathrm{D}} \cos \left(\omega_{\mathrm{d}} t_{\mathrm{k}}+\varphi^{\mathrm{x}}{ }_{\mathrm{d}}\right)\right]
\end{aligned}
$$

Applied substitutions:

- $t_{\mathrm{k}} \cong k \lambda_{\mathrm{r}} /\left(2 v_{\mathrm{m}}\right)$;

- $\varphi_{\sigma_{\mathrm{s}}}=2 \pi \sigma_{\mathrm{s}} v_{\mathrm{m}} T_{\mathrm{D}}$;

- $\omega_{\mathrm{d}} / v_{\mathrm{m}}=2 \pi f_{\mathrm{d}} / v_{\mathrm{m}}=2 \pi \sigma_{\mathrm{d}}, \sigma_{\mathrm{d}}=f_{\mathrm{d}} / v_{\mathrm{m}} \mathrm{k}$.

Developing cosine into Taylor series up to $1^{\text {st }}$ order the Eq. (7) is rewritten as follows:

$$
\begin{aligned}
I_{\sigma_{\mathrm{s}}}\left(x_{\mathrm{k}}\right)=\left\{m_{\mathrm{o}}\left(\sigma_{\mathrm{s}}, u_{o}\right)+a\left(\sigma_{\mathrm{s}}, u_{o}\right) \sigma_{\mathrm{s}} \sin \left[2 \pi \sigma_{\mathrm{d}}\left(\frac{k \lambda_{\mathrm{r}}}{2}\right)+\varphi^{\mathrm{u}}\right]\right. \\
\times I_{\mathrm{s}}\left\{\cos \left[2 \pi \sigma_{\mathrm{s}}\left(\frac{k \lambda_{\mathrm{r}}}{2}\right)+\varphi_{\sigma_{\mathrm{s}}}\right]\right. \\
\left.-2 \pi \sigma_{\mathrm{s}} v_{\mathrm{o}} T_{\mathrm{D}} \sin \left[2 \pi \sigma_{\mathrm{s}}\left(\frac{k \lambda_{\mathrm{r}}}{2}\right)+\varphi_{\sigma_{\mathrm{s}}}\right] \cos \left[2 \pi \sigma_{\mathrm{d}}\left(\frac{k \lambda_{\mathrm{r}}}{2}\right)+\varphi^{\mathrm{x}}{ }_{\mathrm{d}}\right]\right\}
\end{aligned}
$$

Later on the $m_{\mathrm{o}}$ and $a$ coefficients dependence on wavenumber $\sigma_{\mathrm{s}}$ will be neglected. Such an approximation is possible due to the fact that in the ghost component sampling error term is dominant (demonstrated ${ }^{15}$ in the ghost studies on PFS spectra).

Having "2 $2 \pi \sigma_{\mathrm{s}} v_{\mathrm{o}} T_{\mathrm{D}} a$ " as second order infinitesimal factor, the product development goes as follows:

$$
I_{\sigma_{\mathrm{s}}}\left(x_{\mathrm{k}}\right)=m_{\mathrm{o}} I_{\mathrm{s}} \cos \left[2 \pi \sigma_{\mathrm{s}}\left(\frac{k \lambda_{\mathrm{r}}}{2}\right)+\varphi_{\sigma_{\mathrm{s}}}\right]
$$




$$
\begin{gathered}
+a \sigma_{\mathrm{s}} I_{\mathrm{s}} \sin \left[2 \pi \sigma_{\mathrm{d}}\left(\frac{k \lambda_{\mathrm{r}}}{2}\right)+\varphi_{\mathrm{d}}^{u}\right] \cos \left[2 \pi \sigma_{\mathrm{s}}\left(\frac{k \lambda_{\mathrm{r}}}{2}\right)+\varphi_{\sigma_{\mathrm{s}}}\right] \\
-2 \pi \sigma_{\mathrm{s}} v_{\mathrm{o}} T_{\mathrm{D}} m_{\mathrm{o}} I_{\mathrm{s}} \sin \left[2 \pi \sigma_{\mathrm{s}}\left(\frac{k \lambda_{\mathrm{r}}}{2}\right)+\varphi_{\sigma_{\mathrm{s}}}\right] \cos \left[2 \pi \sigma_{\mathrm{d}}\left(\frac{k \lambda_{\mathrm{r}}}{2}\right)+\varphi^{\mathrm{x}}{ }_{\mathrm{d}}\right]
\end{gathered}
$$

Applying substitutions $A_{\mathrm{s}}=m_{\mathrm{o}} I_{\mathrm{s}}, A_{1}=a /\left(2 m_{\mathrm{o}}\right), A_{2}=\pi v_{\mathrm{o}} T_{\mathrm{D}}$ and rewriting Eq. (9) in the wavenumber domain, the measured signal spectrum becomes:

$$
\begin{gathered}
I(\sigma)=A_{\mathrm{s}} \exp \left(i \varphi_{\sigma_{\mathrm{s}}}\right) \delta\left(\sigma-\sigma_{\mathrm{s}}\right) \\
+A_{\mathrm{s}}\left\{A_{1} \sigma_{\mathrm{s}} \exp \left[i\left(\varphi_{\sigma_{\mathrm{s}}}+\varphi^{\mathrm{u}}{ }_{\mathrm{d}}\right)\right]+A_{2} \sigma_{\mathrm{s}} \exp \left[i\left(\varphi_{\sigma_{\mathrm{s}}}+\varphi^{\mathrm{x}}{ }_{\mathrm{d}}+\frac{\pi}{2}\right)\right]\right\} \delta\left[\sigma-\left(\sigma_{\mathrm{s}}+\sigma_{\mathrm{d}}\right)\right] \\
+A_{\mathrm{s}}\left\{A_{1} \sigma_{\mathrm{s}} \exp \left[i\left(\varphi_{\sigma_{\mathrm{s}}}-\varphi^{\mathrm{u}}{ }_{\mathrm{d}}\right)\right]+A_{2} \sigma_{\mathrm{s}} \exp \left[i\left(\varphi_{\sigma_{\mathrm{s}}}-\varphi^{\mathrm{x}}{ }_{\mathrm{d}}+\frac{\pi}{2}\right)\right]\right\} \delta\left[\sigma-\left(\sigma_{\mathrm{s}}-\sigma_{\mathrm{d}}\right)\right]
\end{gathered}
$$

After some mathematical reorganization (and expressing the unknown amplitudes and phases of ghost components as $B$, $C$ and $\left.\varphi_{\sigma \mathrm{B}}, \varphi_{\sigma \mathrm{C}}\right)$ the previous Eq. (10) can be rewritten in the simplified form:

$$
\begin{gathered}
I(\sigma)=A_{\mathrm{s}} \exp \left(i \varphi_{\sigma_{\mathrm{s}}}\right) \delta\left(\sigma-\sigma_{\mathrm{s}}\right)+ \\
{\left[\sigma_{\mathrm{s}} A_{\mathrm{s}} \exp \left(i \varphi_{\sigma_{\mathrm{s}}}\right) \delta\left(\sigma-\sigma_{\mathrm{s}}\right)\right] *\left\{B \exp \left(i \varphi_{\sigma \mathrm{B}}\right) \delta\left[\sigma-\left(+\sigma_{d}\right)\right]+C \exp \left(i \varphi_{\sigma \mathrm{C}}\right) \delta\left[\sigma-\left(-\sigma_{\mathrm{d}}\right)\right]\right\}} \\
=I_{\text {signal }}(\sigma)+\left[\sigma_{\mathrm{s}} I_{\text {signal }}(\sigma)\right] * K_{\sigma_{\mathrm{d}}}(\sigma)
\end{gathered}
$$

where $I_{\text {signl }}(\sigma)$ is the undistorted spectrum and $K_{\sigma \mathrm{d}}(\sigma)$ is the single harmonic vibration kernel, " $\delta$ " and “*” are respectively the Dirac's function and the convolution operator.

For the generic non-monochromatic source of input radiation $I_{\text {continuum }}(\sigma)$ and arbitrary vibration kernel shape $K(\sigma)$, the Eq. (11) can be extended as following:

$$
\begin{aligned}
& I(\sigma)=I_{\text {continuum }}(\sigma)+\left[\sigma I_{\text {continuum }}(\sigma)\right] * K(\sigma) \\
& K(\sigma)=\sum_{j}\left\{B_{j} \exp \left[i \varphi_{\sigma \mathrm{B}_{j}}(\sigma)\right] \delta\left[\sigma-\left(+\sigma_{\mathrm{d}_{j}}\right)\right]\right. \\
& \left.\quad+C_{j} \exp \left[i \varphi_{\sigma \mathrm{C}_{j}}(\sigma)\right] \delta\left[\sigma-\left(-\sigma_{\mathrm{d}_{j}}\right)\right]\right\}
\end{aligned}
$$

\subsection{Vibration effect: Direct Model, PFS data}

Typical PFS raw spectra is given on the Fig. 1.

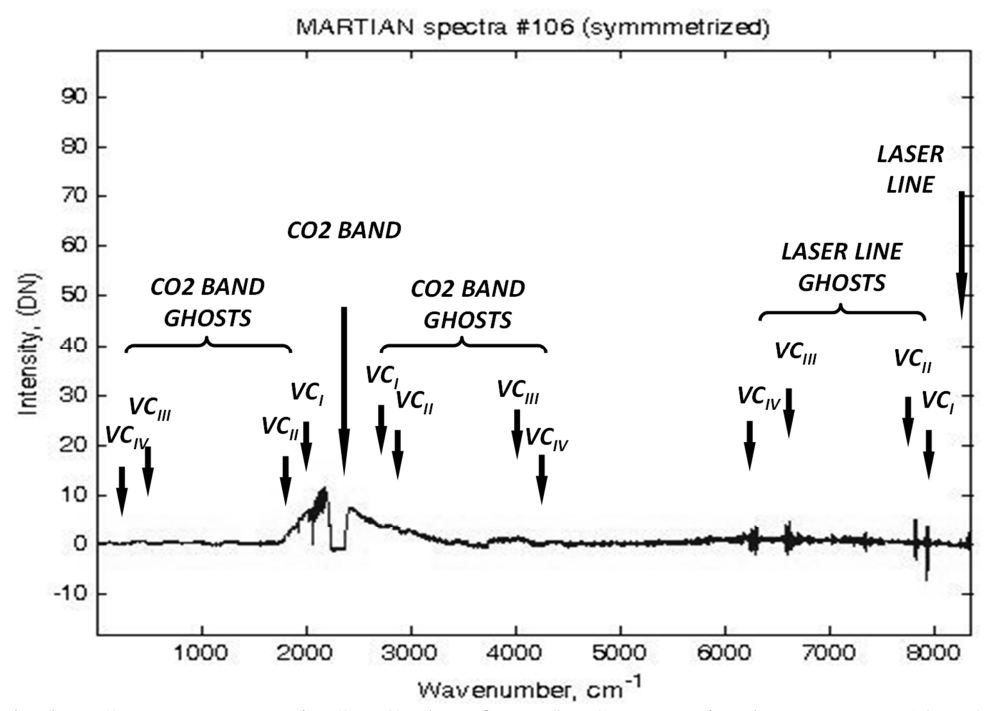

Figure 1. Typical PFS measurement in SWC (data from the Symmetrized spectrum \#106, ORBIT0032). 
It is possible to subdivide the whole spectrum into two parts to be treated separately:

- $\quad$ signal domain in the wavenumber range of 0 to $5000 \mathrm{~cm}^{-1}$;

- laser line domain in the wavenumber range above $5000 \mathrm{~cm}^{-1}$.

The actual energy is present in the signal domain, above the $1700 \mathrm{~cm}^{-1}$, due to the used detector responsivity curve ${ }^{13}$. Below $1700 \mathrm{~cm}^{-1}$ there are only ghosts of the continuum present in the spectrum and no meaningful signal. In the region above $5000 \mathrm{~cm}^{-1}$ the continuum Signal-to-Noise Ratio becomes low due to the strong decrease of the detector responsivity ${ }^{13}$, and thus the laser line domain is characterized mostly by the laser line shape and its ghosts.

In the current research the signal domain was treated only. Actually, after the orbit 662 the SWC reference laser was switched off, leaving the spectrum region above $5000 \mathrm{~cm}^{-1}$ close to the zero value.

Calling back the Eq. (12-13), we rewrite the above expression for the measured raw PFS spectrum $I_{P F S}(\sigma)$, having the input spectrum $I_{\text {Mars }}(\sigma)$ and considering the wavenumber in the second term to be constant, $\sigma \cong \sigma_{\mathrm{k}}=2500 \mathrm{~cm}^{-1}$. Such an approximation is possible due to the fact that the most part of significant signal is actually concentrated in the $2000 . .3000$ $\mathrm{cm}^{-1}$ wavenumber region (Fig. 1). We also include the error term $\xi$, accounting for used approximation in convolution kernel modeling and for other distortions of spectrum, besides the modulation by mechanical vibrations.

$$
\begin{gathered}
I_{P F S}(\sigma)=I_{\text {Mars }}(\sigma) *\left(\delta(\sigma)+\frac{K(\sigma)}{\sigma_{k}}\right)+\xi \\
I_{P F S}(\sigma)=I_{\text {Mars }}(\sigma) * K_{P F S}(\sigma)+\xi \\
K_{P F S}(\sigma)=\delta(\sigma)+B(\sigma) \exp \left[i \varphi_{\sigma \mathrm{B}}(\sigma)\right]+C(\sigma) \exp \left[i \varphi_{\sigma \mathrm{C}}(\sigma)\right]
\end{gathered}
$$

Generally the $B(\sigma)$ and $C(\sigma)$ functions are sparse due to the vibration kernel nature: it is present by a set of separate components, each appearing as the bell-shaped function in the wavenumber domain, although being a pure harmonic in the time domain. This peculiarity is caused by the uneven sampling: because of non-constant mirror speed, the equal OPD step does not correspond to the equal time step. The estimation of those functions will be performed by the inversion procedure described in the next section.

\section{PFS SPECTRA CORRECTION TECHNIQUE THOUGH THE INVERSE PROBLEM SOLUTION}

\subsection{Problem Inversion}

Starting from the direct model, represented by the Eq. (15.2), we proceed to the inversion ${ }^{16}$ of the problem (estimation of the Martian spectrum) through a semi-blind deconvolution method. For that purpose we introduce a Loss Function $L F$, whose minimum would provide the estimates of Martian spectrum $\hat{I}_{\text {Mars }}$ and vibration kernel $\widehat{K}_{\mathrm{PFS}}$. This function not only quantifies the fitting of the estimated data to the original model, but also includes additional terms characterizing the available information about the physical data, such as the sparsity of vibration kernel and smoothness of the Martian spectrum.

$$
\begin{gathered}
\hat{I}_{\mathrm{Mars}}(\sigma), \widehat{K}_{\mathrm{PFS}}(\sigma)=\arg \min _{I_{\mathrm{Mars}}, K_{\mathrm{PFS}}} L F\left(I_{\mathrm{Mars}}, K_{\mathrm{PFS}}\right) \\
L F=\frac{1}{2}\left\|I_{\mathrm{PFS}}-K_{\mathrm{PFS}} * I_{\mathrm{Mars}}\right\|_{2}^{2}+\lambda_{\mathrm{K}}\left\|K_{\mathrm{PFS}}\right\|_{1}+\frac{\lambda_{\mathrm{Mars}}}{2}\left\|D * I_{\mathrm{Mars}}\right\|_{2}^{2}
\end{gathered}
$$

where $\lambda_{\mathrm{K}}$ and $\lambda_{\text {Mars }}$ are the regularization coefficients for the kernel sparsity and spectrum smoothness correspondingly and $D$ is the discrete derivation operator.

\subsection{Iterative procedure for the inverse problem solution}

The solution of the inverse model, represented by the Eq. (16), is performed iteratively, starting from the initial estimates of spectrum $\hat{I}_{\text {Mars }}^{0}$ and kernel $\widehat{K}_{\mathrm{PFS}}^{0}$, and at $\mathrm{n}^{\text {th }}$ iteration determining the estimates $\hat{I}_{\text {Mars }}^{n}$ and $\widehat{K}_{\mathrm{PFS}}^{n}$. The output of algorithm after $\mathrm{N}$ iterations would be the final estimates of the kernel $\widehat{K}_{\mathrm{PFS}}^{N}$ and spectrum $\hat{I}_{\mathrm{Mars}}^{N}$. The iterative process schematically is shown on Fig. 2. 


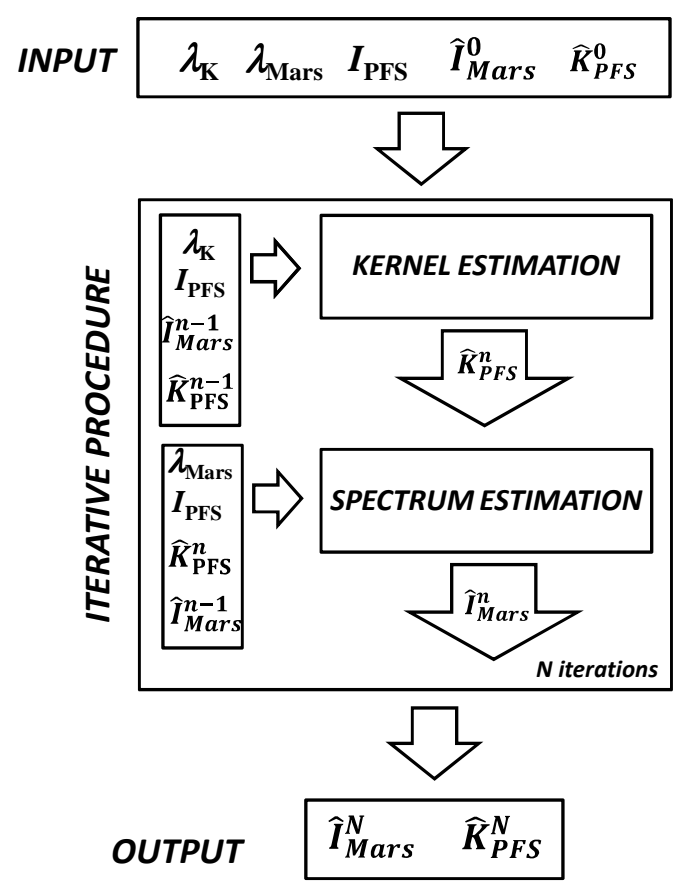

Figure 2. Iterative procedure for the inverse problem solution.

Since the spectra $\hat{I}_{\text {Mars }}^{n}$ is estimated without knowing the kernel $\widehat{K}_{\mathrm{PFS}}^{n}$, the deconvolution is blind. But it has to be noticed, that as the very first step of the proposed algorithm is the kernel estimation $\widehat{K}_{\mathrm{PFS}}^{1}$, then the crucial initial parameter for the efficient algorithm is the initial guess of Martian spectrum $\hat{I}_{\text {Mars }}^{0}$, which has to possess the properties of the real expected solution, otherwise algorithm might converge to some non-physical result. That's why we can qualify this deconvolution as semi-blind. The initialization of kernel $\widehat{K}_{\mathrm{PFS}}^{0}$ instead does not affect the quality of results, but only the algorithm convergence rate.

\subsection{Initialization choices for the semi-blind-deconvolution method}

Vibration Kernel Initialization. As it was mentioned above the quality of kernel initialization provides faster convergence but is not a crucial parameter for the whole algorithm. As an initial guess of kernel for the treated PFS data (Fig. 1: Orbit \#0032, spectrum \#106) we used an approximate kernel estimation from the laser line ghosts by their deconvolution by the laser Dirac in accordance with Eq. (15.2). For the orbits after 662 such an initialization becomes impossible due to the SWC reference laser switching off, and the proposed initialization would be the zero-pattern.

Initial Martian spectrum guess. The Martian spectrum for initial guess estimated by two Planck functions, representing the Martian thermal emission and the solar energy reflected by the Martian surface, with the $\mathrm{CO}_{2}$ absorption band gap in the region of $2200 . .2400 \mathrm{~cm}^{-1}$. The Martian temperature for the first Planck function was estimated from the $2500 . .3000$ $\mathrm{cm}^{-1}$ region of the acquired spectrum, while the second Planck function was scaled to the $3800 . .4200 \mathrm{~cm}^{-1}$ region. Finally to obtain the initial guess of spectrum $\hat{I}_{\text {Mars }}^{0}$ we calibrated data by the known detector responsivity and deep space measurements. ${ }^{13}$

What regards the phase of the initial guess, we took it similar to one of the acquired spectrum in the region of high expected signal, and an extrapolation for the regions of expected ghosts.

To compensate the iterative procedure sensitivity to high frequency noise, both PFS actual data $I_{\mathrm{PFS}}$ and the initial guess $\hat{I}_{\text {Mars }}^{0}$ were filtered with a low-pass filter of cut-off frequency $\frac{1}{20 \Delta \sigma}$, where spectral resolution of acquired data $\Delta \sigma$ is equal to $1.04 \mathrm{~cm}^{-1}$.

The filtered synthetic initial guess of spectrum is shown on Fig. 3 as the comparison with the filtered measured spectrum. 


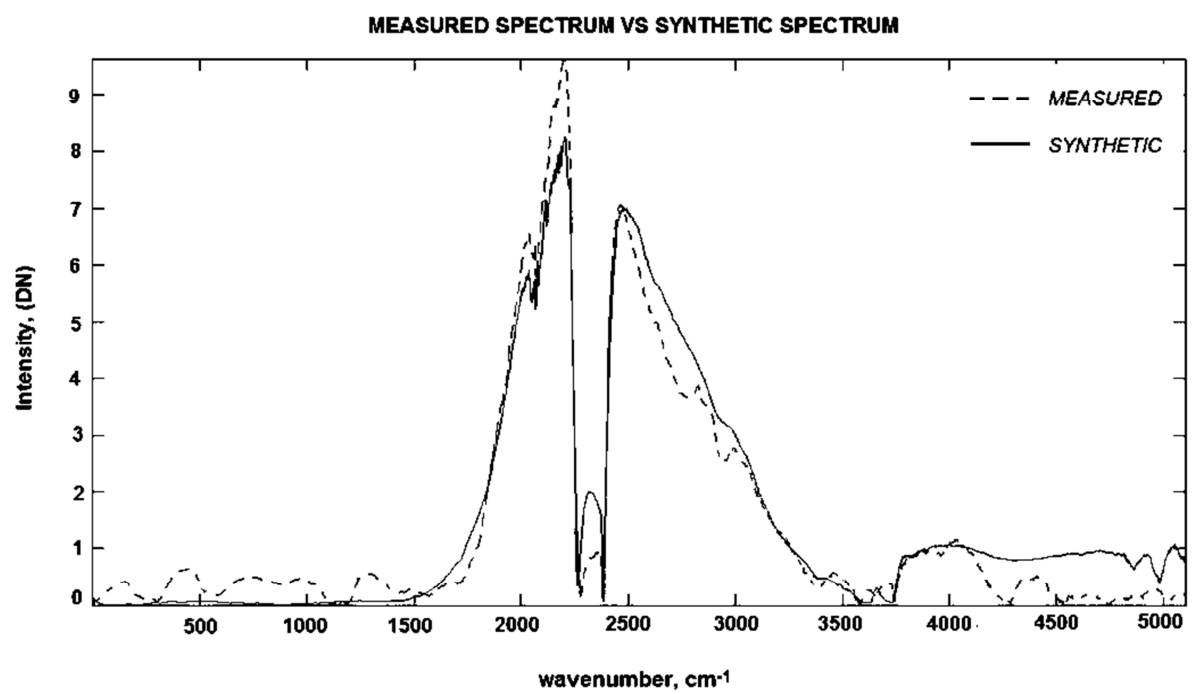

Figure 3. Filtered PFS measured spectrum and the initial guess $\hat{I}_{\text {Mars }}^{0}$.

\subsection{Criteria for result evaluation}

The proposed criteria for the result quality estimation are the following ones:

- distance between acquired PFS spectrum $I_{\mathrm{PFS}}$ and estimated PFS spectrum $\hat{I}_{\mathrm{PFS}}^{N}=\hat{I}_{\mathrm{Mars}}^{N} * \widehat{K}_{\mathrm{PFS}}^{N}$, as if the model is valid the estimate $\hat{I}_{\mathrm{PFS}}^{N}$ has to be as close as possible to $I_{\mathrm{PFS}}$;

- removal of ghosts in the signal domain below $5000 \mathrm{~cm}^{-1}$.

\section{RESULTS}

\subsection{Estimated spectra and vibration kernel}

For Mars spectrum estimation coming from the Orbit \#0032, spectrum \#106, the estimation of signal (after the additional step run on unfiltered data) is shown on the Fig. 4. 

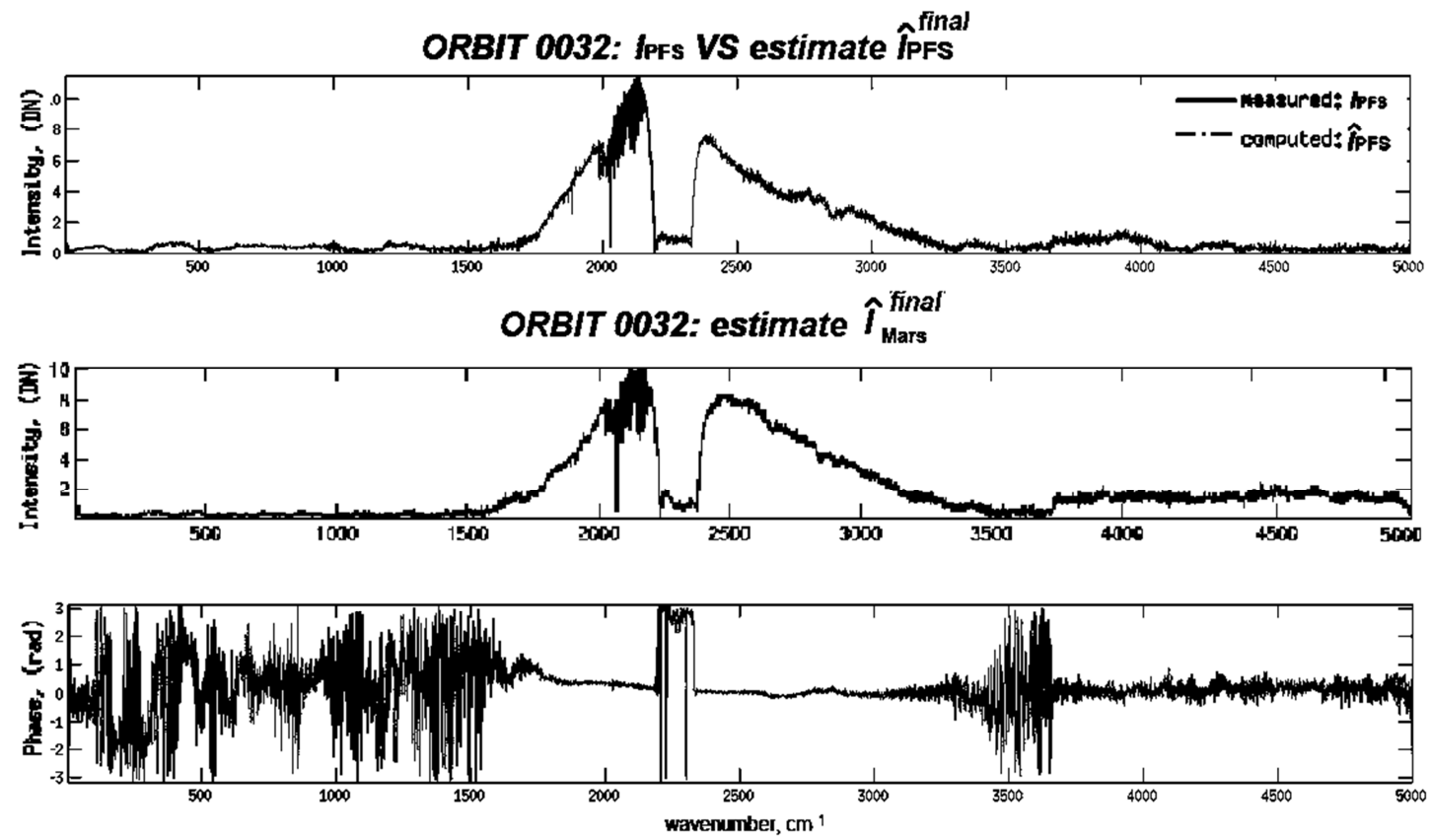

Figure 4. Final results for the spectrum \#106 ORB0032 .

\subsection{Results validation}

As it is seen from Fig. 4, the lack of fit between acquired PFS spectrum $I_{\mathrm{PFS}}$ and the estimated one $\hat{I}_{\mathrm{PFS}}^{\text {final }}=\hat{I}_{\mathrm{Mars}}^{\text {final }}$ * $\widehat{K}_{\mathrm{PFS}}^{\text {final }}$ is very small $\left(\sim 10^{-6}\right)$, proving the solution to agree with actual measured data.

Also the ghost removal in the low wavenumber signal domain (below $1500 \mathrm{~cm}^{-1}$ ) is efficient: the reduction of the energy in the above region is of factor of $\sim 2$.

\section{CONCLUSIONS}

The analytical formulation of modulation effect of mechanical disturbances and the proposed problem inversion makes it possible to perform the correction of the above effect on the PFS data, as well as data coming from any other FTS instrument. Nevertheless, the current approach has some drawbacks:

- the algorithm does not permit to evaluate the high frequency kernel components effect and thus does not perform the correction of narrow spectral features; an improved implementation should correct this phenomena;

- due to the linear assumption of the proposed algorithm, the model treats only part of the information, available from the PFS spectra (i.e. the signal region and not the laser line ghosts one); a non-linear model should be proposed to estimate the vibration kernel and the spectrum through the whole PFS spectral region;

- the implemented iterative procedure is controlled by two regularization parameters and is not optimized in terms of time consumption; robustness of the algorithm shall be improved to be applied on a large dataset.

The next step would be the extension of the direct model also on the laser line region. This will provide the additional criteria for the validation of results: comparison of the evaluated laser line ghost with the actually present ones. Another model modification will be pointed towards the improvement of the algorithm robustness and efficiency, in order to make possible the automatic data treatment procedure. 


\section{REFERENCES}

[1] Saggin, B., Comolli, L. and Formisano. V., "Mechanical disturbances in Fourier spectrometers," Applied Optics 46:5248-56 (2007).

[2] Comolli, L. and Saggin. B., "Evaluation of the sensitivity to mechanical vibrations of an IR Fourier spectrometer," Review of Scientific Instruments 76:123112 (2005);

[3] Toyoshima, M. et al., "Transfer function of microvibrational disturbances on a satellite," 21st International Communications Satellite Systems Conference and Exhibit AIAA 2003-2406 (2003)

[4] Formisano, V. et. al., "The Planetary Fourier Spectrometer (PFS) onboard the European Mars Express mission," Planetary and Space Science 53:963-74 (2005).

[5] Zachor, A. S. and Aaronson, S. M., "Delay compensation: its effect in reducing sampling errors in Fourier spectroscopy," Applied Optics 18:68-75 (1979).

[6] Cohen, D. L., "Performance Degradation of a Michelson Interferometer Due to Random Sampling Errors," Applied Optics 38:139-51 (1999).

[7] Bell, E. E. and Sanderson, R. B., "Spectral Errors Resulting from Random Sampling-Position Errors in Fourier Transform Spectroscopy,” Applied Optics 11:688-9 (1972).

[8] Palchetti, L. and Lastrucci. D., "Spectral noise due to sampling errors in Fourier-transform spectroscopy," Applied Optics 40:3235-43 (2001).

[9] Formisano, V. et. al., "PFS: a Fourier spectrometer for the study of Martian atmosphere," Advances in Space Research 19:1277-80 (1997).

[10]Formisano, V. et. al., "Infrared spectrometer PFS for the Mars94 orbiter," Advances in Space Research 17:61-4 (1996).

[11]Formisano, V. et. al., "The planetary Fourier spectrometer (PFS) for the orbiter of the spacecraft Mars96," Planetary and Space Science 44:889-97 (1996).

[12]Formisano, V. et. al., "PFS for Mars Express: a new approach to study Martian atmosphere," Advances in Space Research 29:131-42 (2002).

[13] Giuranna, M. et al., "Calibration of the Planetary Fourier Spectrometer short wavelength channel," Planetary and Space Science 53:975-91 (2005).

[14] Giuranna, M. et al., "Calibration of the Planetary Fourier Spectrometer long wavelength channel," Planetary and Space Science 53:993-1007 (2005).

[15] Comolli, L., "Mechanical Disturbances in Fourier Spectrometers," PhD Thesis, Politecnico di Milano, (20052006).

[16] Idier, J., [Bayesian Approach to Inverse Problems], ISTE Ltd and John Wiley \& Sons Inc, (2008). 